\title{
MULTI-SOLITON SOLUTIONS OF THE GENERALIZED WEIERSTRASS SYSTEM
}

\author{
P. Bracken* \\ A. M. Grundland ${ }^{\dagger}$
}

CRM-2625

September 1999

\footnotetext{
${ }^{*}$ Centre de recherches mathématiques, Université de Montréal, C.P. 6128 Succ. Centre-Ville, 2910 Chemin de la Tour, Montréal, Québec H3C 3J7 Canada, bracken@crm.umontreal.ca

${ }^{\dagger}$ Centre de recherches mathématiques, Université de Montréal, C.P. 6128 Succ. Centre-Ville, 2910 Chemin de la Tour, Montréal, Québec H3C 3J7 Canada, grundlan@crm. umontreal.ca
} 



\begin{abstract}
The Bäcklund transformation for the generalized Weierstrass system is derived. The permutability theorem for this Bäcklund transformation is formulated and several classes of multi-soliton solutions are obtained through the use of the permutability theorem.
\end{abstract}

\title{
Résumé
}

On dérive la transformation de Bäcklund pour le système de Weierstrass géneralisé. Le théorème de permutabilité de cette transformation de Bäcklund est formulé et plusieurs classes de solutions multisolitoniques sont obtenues en utilisant ce théorème. 



\section{The Generalized Weierstrass System and Associated Sigma Model.}

The generalized Weierstrass (GW) system for inducing constant mean curvature surfaces has been introduced by B. Konopelchenko [1-3]. This system is described by the Dirac type equations

$$
\partial \psi_{1}=p \psi_{2}, \quad \bar{\partial} \psi_{2}=-p \psi_{1}, \quad \text { where } \quad p=\left|\psi_{1}\right|^{2}+\left|\psi_{2}\right|^{2},
$$

and their conjugates, where $\partial=\partial / \partial z$ and $\bar{\partial}=\partial / \partial \bar{z}$.

The system (1) induces a set of constant mean curvature surfaces embedded in $\mathbb{R}^{3}$. These surfaces are obtained by the parametrization $(z, \bar{z}) \rightarrow\left(X_{1}(z, \bar{z}), X_{2}(z, \bar{z}), X_{3}(z, \bar{z})\right)$ such that

$$
\begin{gathered}
X_{1}+i X_{2}=2 i \int_{z_{0}}^{z}\left(\bar{\psi}_{1}^{2} d z^{\prime}-\bar{\psi}_{2}^{2} d \bar{z}^{\prime}\right), \quad X_{1}-i X_{2}=2 i \int_{z_{0}}^{z}\left(\psi_{2}^{2} d z^{\prime}-\psi_{1}^{2} d \bar{z}^{\prime}\right), \\
X_{3}=-2 \int_{z_{0}}^{z}\left(\bar{\psi}_{1} \psi_{2} d z^{\prime}+\psi_{1} \bar{\psi}_{2} d \bar{z}^{\prime}\right) .
\end{gathered}
$$

We start our analysis by considering certain aspects of complete integrability of GW system (1) in the context of a two-dimensional nonlinear sigma model. We define a new complex variable

$$
\rho=\frac{\psi_{1}}{\bar{\psi}_{2}} .
$$

It has been shown $[4,5]$ that if $\psi_{1}$ and $\psi_{2}$ are solutions of GW system (1), then the function $\rho$ defined by (2) is a solution of the Euclidean sigma-model equations

$$
\partial \bar{\partial} \rho-\frac{2 \bar{\rho}}{1+|\rho|^{2}} \partial \rho \bar{\partial} \rho=0, \quad \bar{\partial} \partial \bar{\rho}-\frac{2 \rho}{1+|\rho|^{2}} \bar{\partial} \bar{\rho} \partial \bar{\rho}=0 .
$$

Conversely, if $\rho$ is a solution to (3), then the solutions $\psi_{1}$ and $\psi_{2}$ of GW system (1) take the form

$$
\psi_{1}=\epsilon \rho \frac{(\bar{\partial} \rho)^{1 / 2}}{1+|\rho|^{2}}, \quad \psi_{2}=\epsilon \frac{(\partial \rho)^{1 / 2}}{1+|\rho|^{2}}, \quad \epsilon^{2}=1 .
$$

Formula (4) can be identified with the stereographic coordinate representation of the stationary 2-dimensional Heisenberg model [6]

$$
[S, \partial \bar{\partial} S]=0, \quad S=\left(\begin{array}{cc}
s_{3} & \bar{s}_{+} \\
s_{+} & -s_{3}
\end{array}\right), \quad \operatorname{det} S=-1,
$$

where $S$ is the spin matrix which belongs to the Hermitian space $S U(2) / U(1)$. The matrix $S$ in terms of $\rho$ is given by

$$
S=\frac{1}{1+|\rho|^{2}}\left(\begin{array}{cc}
1-|\rho|^{2} & 2 \bar{\rho} \\
2 \rho & -1+|\rho|^{2}
\end{array}\right), \quad s_{+}=\frac{2 \rho}{1+|\rho|^{2}}, \quad s_{3}=\frac{1-|\rho|^{2}}{1+|\rho|^{2}} .
$$

and in terms of the complex functions $\psi_{1}$ and $\psi_{2}$ by

$$
S=\frac{1}{p}\left(\begin{array}{cc}
-\left|\psi_{1}\right|^{2}+\left|\psi_{2}\right|^{2} & 2 \bar{\psi}_{1} \bar{\psi}_{2} \\
2 \psi_{1} \psi_{2} & \left|\psi_{1}\right|^{2}-\left|\psi_{2}\right|^{2}
\end{array}\right) .
$$

It is well known [7] that the sigma model equations (3) can be obtained from an Euler-Lagrange equation if we take a Lagrangian of the form

$$
\mathcal{L}=\int \frac{\partial \rho \partial \bar{\rho}}{\left(1+|\rho|^{2}\right)^{2}} d z d \bar{z}
$$


Then the Euler-Lagrange equation yields

$$
\bar{\partial} \frac{\delta \mathcal{L}}{\delta(\partial \bar{\rho})}-\frac{\delta \mathcal{L}}{\delta \bar{\rho}}=\frac{1}{\left(1+|\rho|^{2}\right)^{2}}\left(\bar{\partial} \partial \rho-\frac{2 \bar{\rho}}{1+|\rho|^{2}} \partial \rho \bar{\partial} \rho\right)=0 .
$$

This is identically satisfied whenever the sigma model equations (3) hold.

Now, we can derive GW system (1) from Hamiltonian equations. If we define new independent variables $t$ and $x$ by $z=t+i x$, then a real integral of the motion for GW system (1) is independent of $t$ and can be written in the form

$$
\mathcal{H}=\int d s\left[i\left(\psi_{1, s} \bar{\psi}_{2}+\bar{\psi}_{1} \psi_{2, s}\right)+\frac{1}{2}\left(\left|\psi_{1}\right|^{2}+\left|\psi_{2}\right|^{2}\right)^{2}\right]
$$

The quantity $\mathcal{H}$ represents a Hamiltonian since it satisfies the following equations

$$
\psi_{1, t}=\left\{\psi_{1}, \mathcal{H}\right\}, \quad \psi_{2, t}=\left\{\psi_{2}, \mathcal{H}\right\}
$$

where \{\} is the Poisson bracket defined by

$$
\left\{F_{1}, F_{2}\right\}=\int d \tau\left[\left(\frac{\delta F_{1}}{\delta \psi_{1}} \frac{\delta F_{2}}{\delta \bar{\psi}_{2}}-\frac{\delta F_{1}}{\delta \psi_{2}} \frac{\delta F_{2}}{\delta \bar{\psi}_{1}}\right)-\left(\frac{\delta F_{2}}{\delta \psi_{1}} \frac{\delta F_{1}}{\delta \bar{\psi}_{2}}-\frac{\delta F_{2}}{\delta \psi_{2}} \frac{\delta F_{1}}{\delta \bar{\psi}_{1}}\right)\right] .
$$

In order to derive equations (1) explicitly, it is convenient to consider first the case in which $F_{1}=$ $\psi_{1}$ and $F_{2}=\mathcal{H}$. From the condition of linear independence of derivatives $\delta \psi_{1}$ and $\delta \psi_{2}$, after differentiating $\mathcal{H}$ we obtain

$$
\frac{\delta \mathcal{H}}{\delta \bar{\psi}_{2}(x)}=i \psi_{1, \tau}+\left(\left|\psi_{1}\right|^{2}+\left|\psi_{2}\right|^{2}\right) \psi_{2} .
$$

Substituting (8) into (6), we get the first equation of system (1)

$$
\left\{F_{1}, F_{2}\right\}=\left\{\psi_{1}(x), \mathcal{H}\right\}=\int d \tau \delta(\tau-x)\left\{i \psi_{1, \tau}+\left(\left|\psi_{1}\right|^{2}+\left|\psi_{2}\right|^{2}\right) \psi_{2}\right\}=i \psi_{1, x}+\left(\left|\psi_{1}\right|^{2}+\left|\psi_{2}\right|^{2}\right) \psi_{2} .
$$

This procedure can be repeated by taking $F_{1}=\psi_{2}$ and $F_{2}=\mathcal{H}$, and using the second equation in (6), what leads to the second equation in system (1). Thus, we obtain an explicit form of GW system (1) in terms of the independent variables $t$ and $x$

$$
\psi_{1, t}-i \psi_{1, x}=\left(\left|\psi_{1}\right|^{2}+\left|\psi_{2}\right|^{2}\right) \psi_{2}, \quad \psi_{2, t}+i \psi_{2, x}=-\left(\left|\psi_{1}\right|^{2}+\left|\psi_{2}\right|^{2}\right) \psi_{1} .
$$

System (1) admits several conserved quantities [2]. The conservation of current is given by

$$
J=\bar{\psi}_{1} \partial \psi_{2}-\psi_{2} \partial \bar{\psi}_{1}, \quad \bar{\partial} J=0
$$

Note that some classes of solutions to GW system (1) can be obtained directly by applying the transformation (4) to the solutions of the sigma-model equation (3). A simple class of solutions of (1), corresponding to an analytic choice for the function $\rho$, (and to so called splitting solutions of $(3)$, since $\partial \bar{\partial} \rho=0$ ), was discussed in detail in [5]. In this case, the current $J$ in (9) is identically zero. In Section 3, we demonstrate the construction of more complex, multi-soliton solutions for which $\partial \bar{\partial} \rho \neq 0$ holds and consequently, the current $J$ is a nonzero function.

\section{The Auto-BT for the Generalized Weierstrass System.}

In this section we demonstrate a connection between the GW system and the Sh-Gordon type equation, and derive through this link the Auto-Bäcklund transformation (BT). Let us change the 
dependent variables $\psi_{1}$, and $\psi_{2}$ in (1) to the variables $p$ and $J$ given in (1) and (9), respectively. Differentiating $p$ with respect to $z$ and $\bar{z}$, we obtain

$$
\partial p=\psi_{1}\left(\partial \bar{\psi}_{1}\right)+\bar{\psi}_{2}\left(\partial \psi_{2}\right), \quad \bar{\partial} p=\bar{\psi}_{1}\left(\bar{\partial} \psi_{1}\right)+\psi_{2}\left(\bar{\partial} \bar{\psi}_{2}\right), \quad \partial \bar{\partial} p=\bar{\partial} \psi_{1} \partial \bar{\psi}_{1}+\bar{\partial} \psi_{2} \partial \psi_{2}-p^{3} .
$$

It has been shown in [8] that, by using (10) and conservation law (9), GW system (1) can be decoupled into a direct sum of an elliptic Sh-Gordon type equation and Laplace equation

$$
\partial \bar{\partial} \ln p=-p^{2}+\frac{|J|^{2}}{p^{2}}, \quad \bar{\partial} J=0 .
$$

We now apply the conditional symmetry method, developed in [9], to equation (11) in order to construct the BT and to build $N$-soliton solutions. We subject equations (11) to the differential constraints (DCs) of the Riccati form

$$
\partial p=A_{1}^{0}(z, \bar{z})+A_{1}^{1}(z, \bar{z}) p+A_{1}^{2}(z, \bar{z}) p^{2}, \quad \bar{\partial} p=A_{2}^{0}(z, \bar{z})+A_{2}^{1}(z, \bar{z}) p+A_{2}^{2}(z, \bar{z}) p^{2},
$$

where the $A_{j}^{i}$ are some functions to be determined. Then, for any holomorphic function $J$, the overdetermined system composed of (11) and (12) possesses nontrivial solutions of the form

$$
A_{1}^{0}=-\lambda \frac{J}{q}, \quad A_{1}^{1}=\frac{\partial q}{q}, \quad A_{1}^{2}=-\lambda q, \quad A_{2}^{0}=-\frac{q}{\lambda}, \quad A_{2}^{1}=-\frac{\partial q}{q}, \quad A_{2}^{2}=-\frac{\bar{J}}{\lambda q},
$$

where the function $q$ satisfies equation (11) and $\lambda$ is a complex constant. Substituting (13) into (12), we have

$$
\partial p=-\lambda q p^{2}+\frac{\partial q}{q} p-\frac{\lambda J}{q}, \quad \bar{\partial} p=-\frac{\bar{J}}{\lambda q} p^{2}-\frac{\bar{\partial} q}{q} p-\frac{q}{\lambda}, \quad \bar{\partial} J=0 .
$$

Hence, for any holomorphic function $J$, there exists an Auto-BT, defined by first order DC's (14), between the sets of solutions of system (11). The arbitrary complex constant $\lambda$ is called the Bäcklund parameter. Taking $p=\phi_{1} / \phi_{2}$ we get the linear spectral problem with spectral parameter $\mu$

$$
\partial\left(\begin{array}{c}
\phi_{1} \\
\phi_{2}
\end{array}\right)=\left(\begin{array}{cc}
\frac{\partial q}{2 q} & \frac{\mu}{q} \\
-\mu q & -\frac{\partial q}{2 q}
\end{array}\right)\left(\begin{array}{c}
\phi_{1} \\
\phi_{2}
\end{array}\right), \quad \bar{\partial}\left(\begin{array}{c}
\phi_{1} \\
\phi_{2}
\end{array}\right)=\left(\begin{array}{cc}
\frac{\bar{\partial} q}{2 q} & \frac{q}{\mu} \\
-\frac{\bar{J}}{\mu q} & -\frac{\bar{\partial} q}{2 q}
\end{array}\right)\left(\begin{array}{l}
\phi_{1} \\
\phi_{2}
\end{array}\right),
$$

since the compatibility condition for them reproduces the original system (1). Now to see the connection to the modified Sinh-Gordon equation, we introduce the new variable $\varphi=2 \ln p$ in equation (11)

$$
\partial \bar{\partial} \varphi=-2\left(e^{\varphi}-|J|^{2} e^{-\varphi}\right)=-4 \sinh \varphi-2\left(1-|J|^{2}\right) e^{-\varphi}, \quad \bar{\partial} J=0,
$$

and differential constraints (14) become

$$
\partial \varphi=-\lambda e^{\varphi+v}+\partial v-J \lambda e^{-v}, \quad \bar{\partial} \varphi=-\frac{\bar{J}}{\lambda} e^{\varphi-v}-\bar{\partial} v e^{\varphi}-\frac{e^{v}}{\lambda}, \quad \bar{\partial} J=0,
$$

where the function $v$ satisfies (15). 
Now we discuss the permutability theorem [10] for the system (11). The Auto-BT (14) can be written in the form

$$
\partial \ln p=\lambda\left[-p q-\frac{J}{p q}\right]+\partial \ln q, \quad \bar{\partial} \ln p=\frac{1}{\lambda}\left[-\frac{p \bar{J}}{q}-\frac{q}{p}\right]-\bar{\partial} \ln q .
$$

We introduce new dependent variables $y=\ln \frac{p}{J^{1 / 4}}, \hat{y}=\ln \frac{q}{J^{1 / 4}}$. Then, we have,

$$
p q=J^{1 / 2} e^{y+\hat{y}}, \quad y-\hat{y}=\ln \frac{p}{q}, \quad \bar{\partial} \ln p q=\bar{\partial}\left(y+\hat{y}+\frac{1}{2} \ln J\right)
$$

and so, the equations (16) can be written as follows

$$
\partial(y-\hat{y})=-2 \lambda J^{1 / 2} \cosh (y+\hat{y}), \quad \bar{\partial}\left(y+\hat{y}+\frac{1}{2} \ln J\right)=-\frac{2}{\lambda} J^{1 / 2} \cosh \left(y-\hat{y}+\frac{1}{2} \ln J\right) .
$$

Two solutions $\left(y_{0}, y_{1}\right)$ can be linked through a Bäcklund parameter $\lambda_{1}$ using the first equation in (17). The solutions $\left(y_{1}, y_{12}\right)$ can be linked in the same way through another value of the Bäcklund parameter $\lambda_{2}$. Repeating this procedure with the parameters in the reverse order starting from $y_{0}$ we arrive at a solution $y_{21}$. The permutability theorem states that $y_{12}=y_{21}$ which leads to the following relationship between the particular solutions $y_{0}, y_{1}, y_{2}$ and $y_{12}$

$$
\tanh \frac{y_{12}-y_{0}}{2}=\frac{\left(\mu_{1}+\mu_{2}\right)}{\left(\mu_{1}-\mu_{2}\right)} \tanh \frac{y_{1}-y_{2}}{2}, \text { where } \mu_{i}=\lambda_{i} J_{i}^{1 / 2}
$$

\section{Multi-Soliton Solutions.}

We now proceed to construct multi-soliton solutions to GW system (1) based on the nonsplitting class of solutions of (3) and obtained by exploiting the Auto-BT (14). In transformation (18), we take the Bäcklund parameters in the form $\mu_{1}=-\eta+i \xi$ and $\mu_{2}=\eta+i \xi$ with $\left|\mu_{1}\right|^{2}=1$. This choice of parameters leads to a real valued function $p_{12}(z, \bar{z})=\exp \left(J^{1 / 4} y_{12}\right)$ which satisfy equation (11) for a given value of the current $J$ given by (9). So, the functions $\psi_{1}$ and $\psi_{2}$ have to satisfy the overdetermined system composed of GW system (1) and the functional constraint

$$
\left|\psi_{1}\right|^{2}+\left|\psi_{2}\right|^{2}=p_{12}(z, \bar{z}) \in \mathbb{R}
$$

3.1. Algebraic multi-soliton solutions of the GW system. A particular class of rational solutions to the sigma model equation (3) is obtained by taking products of the fundamental solutions of the form

$$
\rho_{j}=\frac{z-a_{j}}{\bar{z}-a_{j}}
$$

where the $a_{j} \in \mathbb{R}$ and $j=1, \cdots, N$. Using transformation (4), we can find the corresponding solutions to GW system (1) and then use the so obtained functions $\psi_{i}$ to determine the expression for $q$. Once we have defined $q$, then, from BT (14), we can calculate multi-soliton solutions. This leads us to the following algebraic multi-soliton solution of the GW system (1)

$$
\psi_{1}=\frac{\epsilon}{2}\left\{\sum_{j=1}^{N} \frac{1}{\left(\bar{z}-a_{j}\right)} \prod_{k=1}^{N} \frac{z-a_{k}}{\bar{z}-a_{k}}\right\}^{1 / 2}, \quad \psi_{2}=\frac{\epsilon}{2}\left\{\sum_{j=1}^{N} \frac{1}{\left(z-a_{j}\right)} \prod_{k=1}^{N} \frac{z-a_{k}}{\bar{z}-a_{k}}\right\}^{1 / 2}, \quad p=\frac{1}{2}\left|\sum_{j=1}^{N} \frac{1}{z-a_{j}}\right| .
$$

Another nonsplitting class of solutions to (1) can be obtained from the following rational solution of the sigma model equation (3)

$$
\rho_{n}=\left(\frac{z-a_{n}}{\bar{z}-a_{n}}\right)^{n}
$$


By exploiting BT (14) we obtain the explicit form of an algebraic multi-soliton solution of GW system (1)

$$
\begin{gathered}
\psi_{1}=\frac{\epsilon}{2}\left[\sum_{j=1}^{N} \frac{j}{\left(\bar{z}-a_{j}\right)} \prod_{k=1}^{N}\left(\frac{z-a_{k}}{\bar{z}-a_{k}}\right)^{k}\right]^{1 / 2}, \quad \psi_{2}=\frac{\epsilon}{2}\left[\sum_{j=1}^{N} \frac{j}{\left(z-a_{j}\right)} \prod_{k=1}^{N}\left(\frac{z-a_{k}}{\bar{z}-a_{k}}\right)^{k}\right]^{1 / 2}, \\
p=\frac{1}{2}\left|\sum_{j=1}^{N} \frac{j}{z-a_{j}}\right| .
\end{gathered}
$$

where $a_{k} \in \mathbb{R}$. Note that both of the above multi-soliton solutions admit $N$ simple poles. Their topological charges are the same

$$
I=\frac{1}{2 \pi i} \int_{C} \frac{1}{p^{2}}\left(|J|^{2}-p^{4}\right) d z d \bar{z}=\epsilon N
$$

3.2. Periodic multi-soliton solutions of GW system. Finally, an interesting class of nonsplitting solutions to the system (1) can be obtained from the following periodic solution to the sigma model equation (3)

$$
\rho_{j}=\exp \left(\cos \left(z-a_{j}\right)-\cos \left(\bar{z}-a_{j}\right)\right), \quad a_{j} \in \mathbb{R} .
$$

The associated periodic multi-soliton solution of GW system (1), obtained through the Auto-BT (14), takes the form

$$
\begin{gathered}
\psi_{1}=\frac{\epsilon}{2}\left\{-\sum_{j=1}^{N} \sin \left(\bar{z}-a_{j}\right) \exp \sum_{j=1}^{N}\left(\cos \left(z-a_{j}\right)-\cos \left(\bar{z}-a_{j}\right)\right)\right\}^{1 / 2} \\
\psi_{2}=\frac{\epsilon}{2}\left\{-\sum_{j=1}^{N} \sin \left(z-a_{j}\right) \exp \sum_{j=1}^{N}\left(\cos \left(z-a_{j}\right)-\cos \left(\bar{z}-a_{j}\right)\right)\right\}^{1 / 2} \\
p=\frac{1}{2}\left|\sum_{j=1}^{N} \sin \left(z-a_{j}\right)\right| .
\end{gathered}
$$

Similar classes of multi-soliton solutions of (1) can be obtained from expression (19) by replacing the cosine function in the $\rho_{j}$ by sine, sinh or cosh functions.

Acknowledgments: This work was supported by research grant from NSERC of Canada and the Fonds FCAR du Gouvernment du Québec. We would like to thank Professors A. Doliwa and W. Schief for helpful discussions on this subject.

\section{References:}

1. B. G. Konopelchenko, Induced surfaces and their integrable dynamics, Stud. Appl. Math., 96, (1996), 9-51.

2. B. G. Konopelchenko and I. A. Taimanov, Constant mean curvature surfaces via an integrable dynamical system, J. Phys A: Math. Gen. 29, (1996), 1261-1265.

3. R. Carrol and B. G. Konopelchenko, Generalized Weierstrass-Enneper inducing, conformal immersions, and gravity, Int. J. Modern Phys. A, 11, N7 (1996), 1183-1216.

4. K. Kenmotsu, Weierstrass Formula for surfaces of prescribed mean curvature, Math. Ann. 245, (1979), 89-99.

5. P. Bracken, A. M. Grundland, L. Martina, The Weierstrass-Enneper System for Constant Mean 
Curvature Surfaces and the Completely Integrable Sigma Model, J. Math. Phys, 40, (1999), 33793403.

6. V. G. Makhankov and O. K. Pashaev, Integrable Pseudospin Models in Condensed Matter, Sov. Sci. Rev. Math. Phys. 9, (1992).

7. R. Ward, Sigma Models in 2+1 Dimensions, in "Harmonic maps and integrable systems", ed. A. Fordy and J. Wood, Vieweg (1994).

8. E. V. Ferapontov and A. M. Grundland, Bäcklund links between different analytic descriptions of constant mean curvature surfaces, CRM-2614, U. de M. (1999).

9. A. M. Grundland, L. Martina and G. Rideau, Partial differential equations with differential constraints in Lecture Notes AMS-CRM 11, Providence, (1997), 135-154.

10. L. Bianchi, Lezioni di Geometria Differenziale, Vol. 1, Enrico Spoerri, Pisa (1922). 